\title{
Vivência de pais com o Método Canguru: revisão integrativa
}

\author{
The experience of fathers with the kangaroo mother care method: an integrative review
}

Thais Rosental Gabriel Lopes ${ }^{1}$, Jovanka Bittencourt Leite de Carvalho ${ }^{1}$, Tássia Regine de Morais Alves ${ }^{1}$, Anderson Brito de Medeiros ${ }^{1}$, Sylvia Silva Oliveira ${ }^{1}$, Francisco Arnoldo Nunes de Miranda ${ }^{1}$

Objetivo: compreender a vivência de pais com o Método Canguru. Métodos: revisão integrativa da literatura, realizada nas bases de dados: Scientific Electronic Library Online, Medical Literature Analysis and Retrieval System Online, Literatura Latino-Americana e do Caribe em Ciências da Saúde, Web of Science e Current Nursing and Allied Health Literature. Resultados: obtiveram-se 732 artigos e sete foram selecionados. Identificou-se que pais vivenciam o Método Canguru com realização e prazer. Ademais, apresentaram dificuldades, medo e insegurança que podem estar associados ao funcionamento e a dispositivos de apoio à vida, utilizados para garantir a sobrevida de prematuros no cuidado relacionado ao distanciamento da figura paterna com neonato. Conclusão: a figura paterna, atualmente, apresenta-se mais participativa e consciente acerca do papel no âmbito familiar e nos cuidados inerentes ao filho prematuro. No entanto, pais se sentem despreparados, mas orgulhosos e esperançosos com evolução clínica de bebês, ao vivenciar o Método Canguru.

Descritores: Pai; Recém-Nascido Prematuro; Método Canguru; Cuidados de Enfermagem; Saúde MaternoInfantil.

Objective: to understand the experience of fathers with the kangaroo mother care method. Methods: an integrative literature review in the following databases: Scientific Electronic Library Online, Medical Literature Analysis and Retrieval System Online, Latin American and Caribbean Health Sciences, Web of Science, Current Nursing and Allied Health Literature. Results: a total of 732 articles were obtained and seven were selected. It was identified that fathers experienced the kangaroo method with fulfillment and pleasure. They presented difficulties, fears and insecurities that may be associated with the functioning and life support devices used to ensure the survival of premature infants, related to the distancing between father and newborn. Conclusion: the father is currently more participative and aware of his role in the family environment and in the care inherent to the premature child. However, despite feeling unprepared, fathers are proud and hopeful with the clinical evolution when experiencing the kangaroo method.

Descriptors: Fathers; Infant, Premature; Kangaroo-Mother Care Method; Nursing Care; Maternal and Child Health.

\footnotetext{
${ }^{1}$ Universidade Federal do Rio Grande do Norte. Natal, RN, Brasil.
} 


\section{Introdução}

A assistência humanizada aos recém-nascidos de baixo peso ao nascer, por meio do Método Canguru, tem se destacado por proporcionar aproximação precoce e contínua do bebê prematuro aos pais e familiares. Os benefícios do uso dessa metodologia vêm demonstrando a importância do cuidado atento, seguro e reconhecendo as particularidades da prematuridade no seio familiar, pois, na maioria das vezes, o nascimento precoce do filho ocorre de forma inesperada, constituindo, assim, desafio familiar ${ }^{(1)}$.

Nas últimas décadas, a prematuridade tem sido a principal causa de morbidade e mortalidade perinatal e neonatal no mundo, principalmente nos países em desenvolvimento. Estes apresentam condições socioeconômicas e culturais desfavoráveis, as quais refletem na assistência à saúde materna e neonatal, configurando-se em importante problema de saúde

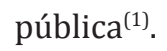

Anualmente, nascem, no mundo, cerca de 20 milhões de crianças prematuras, destas, em torno de um terço vão ao óbito antes de completar o primeiro ano de vida, sendo o peso ao nascer o fator mais significativo dentre as causas de mortalidades neonatais ${ }^{(2)}$. Nas Américas, aproximadamente, $12,0 \%$ do total de neonatos, ou seja, 1.944 .852 recém-nascidos nascem antes de completar 37 semanas de gestação. Consideram-se prematuros extremos quando nascem entre as $22^{\underline{a}}$ e $30^{\underline{a}}$ semanas; e moderados, aqueles com idade gestacional entre as $31^{\underline{a}}$ e $36^{\underline{a}}$ semanas $^{(1)}$.

De forma a modificar esse cenário atual, o Método Canguru integra a agenda de prioridades da política de saúde no Brasil, tendo em vista a exequibilidade. Enquanto medida assistencial, a Atenção Humanizada ao Recém-Nascido de Baixo Peso foi instituída no ano de 2000 e atualizada em 2007, pela Portaria $1.683^{(1)}$, direcionada ao recém-nascido pré-termo e com baixo peso ao nascer, por meio de tecnologias leves e leve-duras, associadas aos cuidados intensivos, os quais ocorrem nos três níveis de assistência à saúde ${ }^{(3)}$.

0 método é desenvolvido em três etapas. A primeira inicia-se no pré-natal de alto risco, seguida da internação do bebê em Unidades de Terapia Intensiva Neonatal e de Cuidado Intermediário Neonatal Convencional, nos quais os pais possuem livre acesso para conhecer e acompanhar os cuidados inerentes ao bebê prematuro. A segunda etapa ocorre em Unidades de Cuidados Intermediários Canguru, local onde os pais passam a ter contato mais próximo e ininterrupto com filhos, o que possibilita a prática da posição canguru, por maior período e frequência, além do empoderamento dos cuidados ao recém-nascido. A terceira acontece no seguimento ambulatorial e ambiente domiciliar, após alta hospitalar da mãe e do filho prematuro, com base em alguns critérios: peso de 2.500 gramas do recém-nascido, além do acompanhamento pela Atenção Básica, com ênfase nas normas do crescimento e desenvolvimento infantil ${ }^{(1,3)}$.

Durante as etapas que contemplam o Método Canguru, pais e familiares devem ser acompanhados por profissionais de saúde capacitados, de forma a assegurar a eficácia do método, bem como a segurança do bebê prematuro, além de motivar o cuidado neonatal ${ }^{(3)}$. Assim, inicialmente, os pais são convidados a realizarem contato pele a pele gradual, permitindo aproximação crescente entre ambos ${ }^{(4)}$.

As normas ministeriais associaram a figura materna à prática do canguru, fato relacionado à importância da amamentação e dos cuidados primários ao bebê prematuro. Já os pais têm se mostrado participativos em diversos aspectos da vida do filho. A presença destes representa o elo entre a genitora e a família, durante o período de internação da díade mãe-filho, revelando-lhes como apoiador domiciliar. 0 cuidado compartilhado contribui significativamente para o desenvolvimento infantil e ameniza a rotina diária da mulher-mã $\mathrm{e}^{(3-4)}$. Adicionam-se a esses aspectos as adaptações familiares com a nova rotina, bem como as tomadas de decisões, as quais envolvem os membros da família de recém-nascidos prematuros ${ }^{(4)}$.

No entanto, estudos encontrados na literatura sobre a vivência de pais nos cuidados aos recém-nascidos prematuros no Método Canguru ainda é restrita, 
tendo o foco das pesquisas direcionado à figura materna, fato associado à importância da mãe nos cuidados ao filho, principalmente na segunda fase, pois, nesse período, a mãe permanece acompanhando o bebê nos cuidados em Unidades de Cuidados Intermediários Canguru. Pesquisas internacionais demonstraram a necessidade de inserir e encorajar pais neste processo $^{(4)}$. Nesse contexto, o Método promove a participação e empoderamento dos pais sobre suas responsabilidades nos cuidados com o seu filho, e mais confiantes para atender as necessidades individuais do prematuro $^{(1)}$.

Dessa forma, diante da relevância da temática e reconhecendo os benefícios da presença paterna no Método Canguru, o suporte emocional à mãe e ao filho e a importância do acompanhamento de pais no desenvolvimento e crescimento infantil, objetivou-se compreender a vivência de pais com o Método Canguru.

\section{Métodos}

Revisão integrativa de literatura, realizada de forma sintetizada, através da coleta de dados de estudos, de maneira abrangente e sistemática. Desta maneira, possibilita conclusões abrangentes sobre o fenômeno estudado, com vistas à compreensão deste, bem como aplicabilidade na prática, baseada em conhecimento científico ${ }^{(5)}$.

Para elaboração de trabalho dessa natureza, faz-se necessário seguir seis etapas: elaboração da pergunta norteadora; busca na literatura; coleta de dados; análise crítica dos estudos incluídos; discussão dos resultados; e apresentação da revisão integrativa ${ }^{(5)}$. Estas etapas foram primeiramente pautadas na identificação do tema e formulação da questão de pesquisa: como pais de filhos prematuros vivenciam o Método Canguru?

A elaboração da questão de pesquisa foi fundamentada na estratégia PICO, acrônimo que corresponde à Paciente, Intervenção, Comparação e Outcomes (desfecho) ${ }^{(6)}$. Desta forma, o P: pais; o I: método canguru; C: ausente; O: vivência. Não houve preenchimento do C, representando a comparação, uma vez que não foi objetivo do estudo.

A etapa de seleção dos estudos envolveu leitura criteriosa dos títulos e resumos e, posteriormente, do texto na íntegra. Foram estabelecidos os critérios de inclusão: artigos originais e de revisões que contemplassem o objetivo do presente estudo e a questão de pesquisa, nos últimos 39 anos (1980-2019). Justifica-se a escolha desse recorte temporal, visto que a prática do Método Canguru foi instituída em 1979. Excluíram-se teses e dissertações, textos incompletos, artigos editoriais, resumos, ou publicações fora do período estabelecido, bem como aquelas que não respondiam à questão de pesquisa.

A extração dos dados ocorreu a partir de criteriosa avaliação, com vistas à leitura interpretativa/ exploratória dos artigos, os quais foram alocados por meio de instrumento adaptado ${ }^{(7)}$, contendo as seguintes informações: identificação do artigo (título, base de dados, periódico, autores e idioma de publicação); dados relativos à pesquisa (delineamento do estudo, objetivo, resultados e conclusões).

A etapa de estratégia de busca ocorreu no acervo disponibilizado, por meio do portal de periódicos da Coordenação de Aperfeiçoamento de Pessoal de Nível Superior, pela Universidade Federal do Rio Grande do Norte, nas seguintes bases de dados: Scientific Electronic Library Online (SCIELO), Medical Literature Analysis and Retrieval System Online (MEDLINE), Literatura Latino-Americana e do Caribe em Ciências da Saúde (LILACS), Web of Science e Current Nursing and Allied Health Literature (CINAHL). O levantamento de artigos ocorreu entre abril e maio de 2019, realizado por dois pesquisadores.

Na fase de elaboração da revisão, foram definidos os descritores a serem utilizados durante a pesquisa: Pai, Recém-Nascido Prematuro e Método Canguru, atrelados por meio do uso do operador booleano AND: Fathers (1\#); Infant, Premature (2\#); Kangaroo-Mother Care Method (3\#). Assim, após o cruzamento de $1 \#$ and $2 \#$ and $3 \#$, foram encontrados 14 artigos; 
$1 \#$ and 3\#, 36; e 1\# and 2\#, encontrados 682, resultando em 732 estudos. Após avaliar os dados duplicados e removidos, a primeira seleção contou com 393 artigos (Figura 1). Destes, realizou-se seleção a partir da análise minuciosa de títulos e resumos, o que resultou em 54 estudos.

A partir da leitura detalhada, foram excluídos 47 estudos, por não contemplarem a questão de pes- quisa. Os artigos selecionados foram sistematicamente analisados e definidos, de forma a compor a amostra final da revisão integrativa de literatura, os quais foram categorizados e interpretados, baseados na literatura pertinente e atualizada. Para minimizar vieses, dois pesquisadores realizaram a leitura dos artigos e preencheram o instrumento de coleta de dados, independentemente.
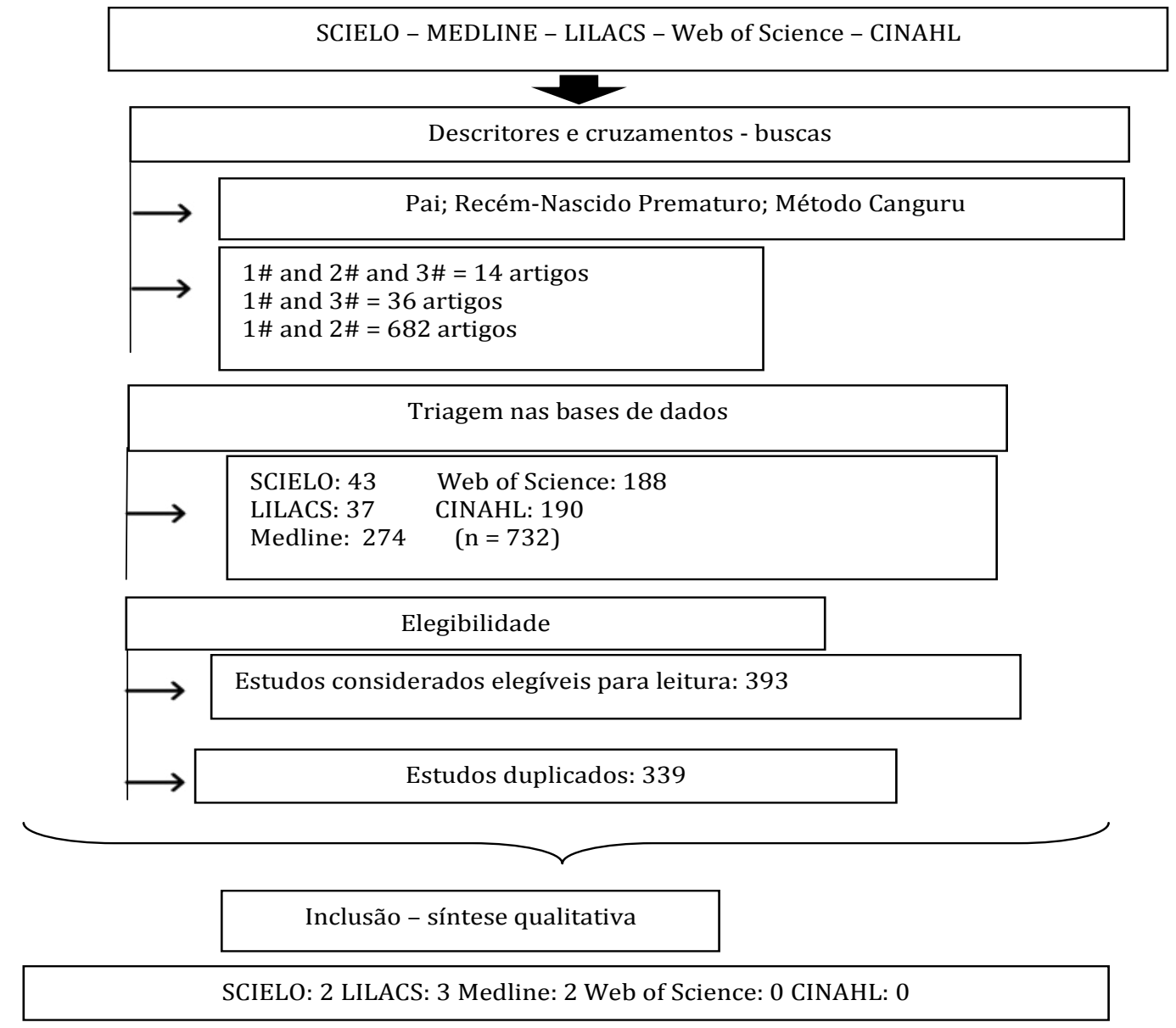

Figura 1 - Fluxograma da seleção de busca, triagem, elegibilidade e definição dos estudos

\section{Resultados}

Referente ao ano de publicação, houve predomínio de publicações no período de 2007 a 2015. A Figura 2 apresenta o quantitativo dos artigos, após aplicar os critérios de inclusão e exclusão da pesquisa. Não foram selecionados estudos nas bases de dados CINAHL e Web of Science.
Quanto ao tipo de estudo, obteve-se maior quantitativo de estudos exploratórios e descritivos, em maioria, em periódicos de língua estrangeira, mais especificamente, na Suécia, no Brasil, em Portugal e na Espanha. Destes, as áreas de Medicina e Enfermagem apresentaram maior quantitativo. 
Após análise dos artigos da revisão de literatura, emergiram-se duas categorias temáticas a partir das áreas predominantes, as quais foram denominadas de: Cuidado paterno com filho prematuro no Mé- todo Canguru, correspondente aos estudos A, B, C, D, E, F e G; e Dificuldades do pai em realizar o Método Canguru, relacionados aos estudos A, B e G.

\begin{tabular}{|l|l|l|}
\hline Id/Autores & \multicolumn{1}{|c|}{ Título do artigo } & \multicolumn{1}{|c|}{ Resultados } \\
\hline $\begin{array}{l}\mathrm{A}^{(8)} \\
\text { Tronchin DMR, } \\
\text { Tsunechiro MA }\end{array}$ & $\begin{array}{l}\text { Cuidar e o conviver com o filho prematuro: a experi- } \\
\text { ência do pai }\end{array}$ & $\begin{array}{l}\text { Medo e preocupações com parto prematuro e felicidade em } \\
\text { estar com filho }\end{array}$ \\
\hline $\begin{array}{l}\mathrm{B}^{(9)} \\
\text { Lundqvist P, et al }\end{array}$ & $\begin{array}{l}\text { From distance toward proximity: fathers' lived expe- } \\
\text { rience of caring for their preterm infants }\end{array}$ & $\begin{array}{l}\text { O papel da equipe de saúde: incentivar e orientar o pai nos } \\
\text { cuidados com filho }\end{array}$ \\
\hline $\begin{array}{l}\mathrm{C}^{(10)} \\
\text { Lindberg B, et al }\end{array}$ & $\begin{array}{l}\text { Adjusting to being a father to an infant born } \\
\text { prematurely: experiences from Swedish fathers }\end{array}$ & $\begin{array}{l}\text { O pai aprende a cuidar dos filhos com auxílio dos profissionais } \\
\text { de saúde }\end{array}$ \\
\hline $\begin{array}{l}\mathrm{D}^{(11)} \\
\text { Blomqvist YT, et al }\end{array}$ & $\begin{array}{l}\text { Kangaroo Mother Care helps fathers of preterm in- } \\
\text { fants gain confidence in the paternal role }\end{array}$ & $\begin{array}{l}\text { Oportunidade de o pai lidar com a prematuridade e desenvol- } \\
\text { ver competências parenterais no Método Canguru }\end{array}$ \\
\hline $\begin{array}{l}\mathrm{E}^{(12)} \\
\text { Gutiérrez JJB }\end{array}$ & $\begin{array}{l}\text { The role of fathers in the postpartum period: experi- } \\
\text { ences with skin to skin method }\end{array}$ & $\begin{array}{l}\text { Incentivo dos profissionais de saúde para o cuidado do pai } \\
\text { com o filho }\end{array}$ \\
\hline $\begin{array}{l}\mathrm{F}^{(13)} \\
\text { Santos ND, et al }\end{array}$ & $\begin{array}{l}\text { Re)cognizing the masculine participation in kanga- } \\
\text { tice of nursing }\end{array}$ & $\begin{array}{l}\text { Bonding and prematurity: exploratory study on early } \\
\text { paternal involvement in hospitalization context } \\
\text { pai sente medo da morte do filho }\end{array}$ \\
\hline $\begin{array}{l}\mathrm{G}^{(14)} \\
\text { Leal I, et al }\end{array}$ & $\begin{array}{l}\text { O receio do pai no primeiro contato com o recém-nascido e } \\
\text { aproximação deste com filho }\end{array}$ \\
\hline
\end{tabular}

Figura 2 - Caracterização dos artigos, conforme identificação, autores, título do artigo e resultados

\section{Discussão}

Esta revisão apresentou como limitação a escassez de estudos, mediante os critérios adotados, sobre a temática discutida, principalmente, das publicações em bases de dados internacionais, com ênfase nas áreas de Medicina e Enfermagem. Este fato demonstra a carência de estudos no âmbito da Enfermagem Neonatal que abordem a vivência de pais no Método Canguru.

A primeira categoria dos resultados se refere ao cuidado paterno com filho prematuro no Método Canguru. Ao se dispor em cuidar do filho e lidar com as particularidades da prematuridade, pode-se evocar no pai que cuida diversos sentimentos antagônicos. 0 banho do bebê prematuro, a troca de fraldas, a alimentação, assim como a posição canguru, desvelando um novo universo ao pai, o que pode gerar sentimentos antagônicos. As preocupações e os medos podem ser percebidas quando associadas ao prazer e alegria de estar junto ao filho que se apresenta antecipa- damente à família ${ }^{(15)}$. 0 período gestacional pode ser compreendido como momento de preparação familiar, seja financeira ou psicológica.

Pesquisa realizada sobre essa temática corrobora com as afirmações anteriores ao revelarem que a relação do pai com o filho se inicia antes da concepção, pois os homens criam expectativas e anseios acerca dessa relação ${ }^{(16)}$. Desta forma, aponta-se a importância da participação do homem durante o pré-natal da companheira, com ênfase no esclarecimento de dúvidas e redução de anseios e expectativas da gestação de pais, associada à possibilidade do nascimento prematuro do filho.

0 período gestacional pode despertar no pai o primeiro desejo de aproximação com o filho, o que ocorre por meio do diálogo com o bebê e do toque sobre o abdômen materno. A proximidade é interrompida com o parto prematuro, informação evidenciada em estudo realizado sobre a relação pai e filho, em 
que se revelou que a interrupção da gestação colabora para afastamento de ambos, pois, após o nascimento, o recém-nascido prematuro necessita de cuidados específicos e, portanto, de internação hospitalar ${ }^{(17)}$.

Nessas circunstâncias, o Método Canguru oportuniza o contato diário com o bebê prematuro, o que propicia a adaptação à nova rotina necessária para assisti-lo. A interação precoce estimula a relação entre o pai e a criança, além de permitir a sensação de ser reconhecido pelo filho ${ }^{(11)}$. Soma-se a tais sentimentos a realização dos pais em praticar o método, além do relaxamento de ambos, proporcionado pelo contato pele a pele ${ }^{(18-19)}$.

Estudos que compuseram esta revisão(8,10-14) contemplam o contentamento paterno em realizar os cuidados com o filho prematuro, por meio do Método Canguru, desencadeando o desejo de estar com o filho e a vontade de exercer a paternidade. Corrobora-se com este achado, no que tange à alegria paterna em estar com filho prematuro, o desejo de acompanhar o desenvolvimento e a evolução clínica diária dos filhos no período de internação ${ }^{(17,20)}$.

No entanto, quatro pesquisas ${ }^{(8,9,11-14)}$ ressaltaram sentimentos ambíguos, como inseguranças, preocupações e medos. No âmbito hospitalar, o medo é algo comum e surge a partir do desconhecimento paterno sobre o ambiente, a impossibilidade de controlar ou mudar as situações vivenciadas junto ao filho, devido às limitações impostas pela prematuridade. Estudo confirma tais dados, ao demonstrar que o pai se sente impotente diante dos procedimentos e da assistência direcionada ao filho ${ }^{(14)}$.

Os primeiros contatos com o filho prematuro no Método Canguru são demonstrados com temor ao toque, associado à fragilidade física e fisiológica. Nessas ocasiões, o pai exprime emoções contraditórias, como fuga da realidade e alegria da aproximação diária com filho ${ }^{(21)}$. Acredita-se que, conforme preconizado pelo Ministério da Saúde do Brasil, o Método Canguru deve ser realizado paulatinamente, respeitando a vontade do pai em estar com o filho, com vistas a superar o medo de causar algum dano físico ao bebê $\hat{}^{(3)}$.
Assim, destaca-se a relevância da assistência dos profissionais de saúde ao pai do pré-termo e a inserção daquele no contexto do cuidado. Esses ambientes compreendem a primeira fase do Método Cangu$\mathrm{ru}$, momento em que ocorrem os primeiros contatos entre pai e filho. Desta forma, por motivos diversos, as unidades descritas podem causar sentimentos adversos no pai, como insegurança quanto à evolução, associada ao medo da morte do filho prematuro.

Frente a isso, esse setor representa o distanciamento do filho com os pais, conforme relatado por estudo que se refere à barreira física imposta pelos aparatos tecnológicos indispensáveis, comuns em Unidades de Tratamento Intensivo Neonatal. Pesquisa descreve que os pais de crianças assistidas nesse espaço caracterizam-no como hostil e estressante, principalmente devido ao fato de ser repleto de aparelhos específicos e procedimentos invasivos ${ }^{(22)}$.

Entende-se que, nos primeiros momentos, o pai se assusta com o filho, fato associado à fragilidade e dimensões corporais diminuídas do bebê. Estudos demonstram que os pais associam o aspecto físico do filho à dificuldade de realizar o Método Canguru e à superproteção ${ }^{(8,13-15)}$. Os obstáculos podem ser minimizados pela equipe de saúde, principalmente a de Enfermagem, na promoção da aproximação precoce entre a família dos recém-nascidos, bem como na inclusão desta nos cuidados, a exemplo da troca de fraldas e da alimentação. Estas atitudes podem proporcionar ao pai o sentimento de responsabilidade com o filho prematuro ${ }^{(23)}$.

A segunda categoria dos resultados faz referência às dificuldades relatadas pelos pais para realização do Método Canguru e adaptação desse bebê à vida extrauterina. A aproximação diária do pai com filho, principalmente nas duas primeiras fases do método, requer adaptação do contexto de vida do pai, que permanece com as atividades habituais. Desta forma, a disponibilidade de horários constituiu uma das barreiras mais comuns enfrentadas pelos homens, relacionadas às atividades laborais e ao suporte à família durante o período de internamento da companheira e 
do filho. Logo, estudos indicaram que os pais se apresentam mais influentes no cuidado ao filho, e preocupando-se em promover carinho e apoiar a companheira na assistência ${ }^{(4,24)}$.

Diante de tal realidade, faz-se necessário que os profissionais de saúde reconheçam o contexto pessoal de pais que acompanham bebês prematuros, enaltecendo as potencialidades e adequando as dificuldades apresentadas. No contexto de cuidados neonatais, os profissionais envolvidos representam, em diversas situações, elo entre o pai e o filho, para que seja possível a contemplação dos cuidados inerentes à prematuridade com segurança e, assim, determinar o empoderamento dos cuidados após a alta hospitalar da díade mãe-filho, para continuidade da terceira fase do Método Canguru.

Dessa forma, a equipe de saúde possui papel ímpar, no que tange à assistência materna e neonatal diante da prematuridade. Esse momento compreende evento inesperado pela família do recém-nascido e envolve mudanças na dinâmica familiar, conforme destacada em estudos ${ }^{(8-9)}$. 0 futuro ainda incerto do bebê que nasce prematuramente gera expectativas e insegurança com relação ao prognóstico ${ }^{(23)}$.

0 período em que o prematuro e a mãe permanecem na Unidade de Cuidados Intermediários Canguru é desconhecido, podendo perdurar de semanas a meses. A necessidade da presença da mãe a distância da rotina pessoal, dos familiares e, em muitos casos, dos outros filhos. Nessa circunstância, o pai representa apoio significativo e tende a minimizar a ansiedade e preocupações maternas. Ademais, os momentos em que o pai permanece junto à companheira e ao filho, no Método Canguru, podem propiciar descanso materno e reduzir o período que permanece institucionalizada junto ao filho.

No âmbito dos cuidados destinados ao recém-nascido prematuro no Método Canguru, estes devem ser realizados de maneira apropriada, pois a forma como o bebê é manuseado pode afetar a estabilidade fisiológica e comportamental, devido à imaturidade morfológica e fisiológica, necessitando, por isso, de cuidado individualizado $^{(16)}$. De modo geral, o homem necessita participar da assistência ao filho, com vistas a não sentir excludentes e incapacitados no ato de cuidar, sentimentos que devem ser minimizados por profissionais e da companheira ${ }^{(17)}$.

Assim, recomenda-se que a família seja acompanhada e orientada por equipe qualificada, com vistas à assistência integral às necessidades do bebê, proporcionando cuidado adequado e, por conseguinte, desenvolvimento crescente e seguro ${ }^{(25)}$.

\section{Conclusão}

Identificou-se que pais, atualmente, apresentam-se mais participativos e conscientes acerca do papel no âmbito familiar e nos cuidados inerentes aos filhos prematuros. No entanto, sentem-se despreparados, mas, orgulhosos e esperançosos com a evolução clínica destes. Compreende-se que o acompanhamento diário, o aumento gradual de peso e cada progresso desencadeiam a esperança da alta hospitalar ao vivenciar a paternidade no Método Canguru.

\section{Colaborações}

Lopes TRG, Carvalho JBL, Alves TRMA, Medeiros $\mathrm{AB}$, Oliveira SS e Miranda FAN contribuíram na concepção e projeto, análise e interpretação dos dados, redação do artigo, revisão crítica relevante do conteúdo intelectual e aprovação final da versão a ser publicada.

\section{Referências}

1. Ministério da Saúde. Secretaria de Atenção à Saúde. Atenção humanizada ao recém-nascido de baixo peso: Método Canguru [Internet]. 2014 [citado 2019 mai 27]. Disponível em: http:// bvsms.saude.gov.br/bvs/publicacoes/metodo_ canguru_manual_tecnico_2ed.pdf

2. Araújo LA, Reis AT. Enfermagem na prática materno-neonatal. Rio de Janeiro: Guanabara Koogan; 2012. 
3. Mantelli GV, Strapasson MR, Pierotto AA, Renosto JM, Silva JF. Método Canguru: percepções da equipe de enfermagem em terapia intensiva neonatal. Rev Enferm UFSM. 2017; 7(1):51-60. doi: https://doi.org/10.5902/2179769221182

4. Shorey S, He HG, Morelius E. Skin-to-skin contact by fathers and the impact on infant and paternal outcomes: an integrative review. Midwifery. 2016; 40(2):207-17. doi: https://doi.org/10.1016/j. midw.2016.07.007

5. Mendes KDS, Silveira RCCP, Galvão CM. Revisão Integrativa: método de pesquisa para a incorporação de evidências na saúde e na enfermagem. Texto Contexto Enferm. 2008; 17(4):758-64. doi: doi.org/10.1590/S0104-07072008000400018

6. Chicharro SCR, Florência MV, Alves SZSP, Andrade M, Valente GSC. Factors facilitating the teachinglearninginnursingeducation:anintegrativereview. Rev Pesqui Cuid Fundam Online. 2016; 8(2):4099108. doi: https://10.9789/2175-5361.2016. v8i2.4099-4108

7. Souza V, Zeitoun SS, Barros ALBL. Decreased cardiac output: a systematic review of the defining characteristics. Acta Paul Enferm. 2011; 24(1):114-9. doi: http://dx.doi.org/10.1590/ S0103-21002011000100017

8. Tronchin DMR, Tsunechiro MA. Cuidar e o conviver com o filho prematuro: a experiência do pai. Rev Latino-Am Enfermagem. 2006; 14(1):93101. doi: https://dx.doi.org/10.1590/S010411692006000100013

9. Lundqvist P, Westas LH, Hallström I. From distance toward proximity: fathers' lived experience of caring for their preterm infants. J Pedriat Nurs. 2007; 2(6):490-7. doi: https://doi.org/10.1016/j. pedn.2007.04.008

10. Lindberg B, Axelsson K, Öhrling K. Adjusting to being a father to an infant born prematurely: experiences from Swedish fathers. Scand J Carring Sci. 2008; 22(1):79-85. doi: https://doi.org/10.1111/ j.1471-6712.2007.00563.x

11. Blomqvist YT, Rubertsson C, Kylberg E, Joreskog K, Nyqvist, KH. Kangaroo Mother Care helps fathers of preterm infants gain confidence in the paternal role. J Adv Nurs. 2011; 68(9):1988-96. doi: doi.org/10.1111/j.1365-2648.2011.05886.x
12. Gutiérrez JJB. The role of fathers in the postpartum period: experiences with skin to skin method. Acta Paul Enferm. 2012; 25(6):914-20. doi: http://dx. doi.org/10.1590/S0103-21002012000600014

13. Santos ND, Machado MED, Christoffel M. (Re) cognizing the masculine participation in kangaroo method: an interface with the assistencial practice of nursing. Cienc Cuid Saúde. 2013; 12(3):461-8. doi: http://dx.doi.org/10.4025/cienccuidsaude. v12i3.11722

14. Leal I, Saldanha N, Oliveira RV. Bonding and prematurity: exploratory study on early paternal involvement in hospitalization context. Psicol Saúde Doenças. 2014; 15(2):454-67. doi: http://dx.doi. org/10.15309/14psd150210

15. Medeiros FB, Piccinini CA. Relação pai-bebê no contexto da prematuridade: gestação, internação do bebê e terceiro mês após a alta hospitalar. Est Psicol. 2015; 32(3):475-85. doi: http://dx.doi. org/10.1590/0103-166X2015000300012

16. Chan GJ, Labar A, Wall S, Atun R. Kangaroo mother care: a systematic review of barriers and enablers. Bull World Health Organ. 2016; 94(2):130-41. doi: https://10.2471/BLT.15.157818

17. Jesus NC, Vieira BDG, Alves VH, Rodrigues DP, Souza RMPS, Paiva ED. The experience of the kangaroo method: the perception of the father. Rev Enferm UFPE on line. 2015; 9(7):854250. doi: https://doi.org/10.5205/1981-8963v9i7a10626p8542-8550-2015

18. Hagen IH, Iversen VC, Svindseth MF. Differences and similarities between mother and fathers of premature chidren: a qualitative study of parent's coping experiences in a neonatal intensive care unit. BMC Pediatric. 2016; 6(92):1-9. doi:https:// doi.org/10.1186/s12887-016-0631-9

19. Medeiros JS, Mascarenhas MFPT. Banho humanizado em recém-nascidos prematuros de baixo peso em uma enfermaria canguru. Rev Ter Ocup Univ. 2010; 21(1):51-60. doi: https://doi. org/10.11606/issn.2238-6149.v21i1p51-60

20. Brito RS, Enders BC. Interacionismo simbólico como perspectiva metodológica. In: Brito RS. Quatro fases do homem no contexto da reprodução. Natal: Observatório RH NESC/UFRN; 2011. 
21. Tamez RN, Silva MJP. Enfermagem na UTI neonatal. Assistência ao recém-nascido de alto risco. Rio de Janeiro: Guanabara Koogan; 2013.

22. Veronez M, Borghesan NAB, Corrêa DAM, Higarashi IH. Experience of mothers of premature babies from birth to discharge: notes of field journals. Rev Gaúcha Enferm. 2017; 38(2):109-19. doi: http://org/10.1590/1983-1447.2017.02.60911

23. Roseiro CP, Paula KMP. Concepções de humanização de profissionais em Unidades de Terapia Intensiva Neonatal. Est Psicol. 2015; 32(1):10919.doi:http://dx.doi.org/10.1590/0103166X2015000100010
24. Souza ML, Silva VCE, Parada CMGR, Zani AV. Repercussões no cuidado domiciliar para o pai participante do protocolo de assistência ao filho prematuro. Rev Eletr Acervo Saúde. 2018; 10(4):1727-34. doi: http://dx.doi.org/10.25248/ REAS227_2018

25. Aires LCP, Santos EKAD, Costa R, Borck M, Custódio ZAD. Baby follow-up in primary care: interface with the third stage of the kangaroo method. Rev Gaúcha Enferm. 2015; 2(spe):224-32. doi: http:// dx.doi.org/10.1590/1983-1447.2015.esp.56805 